\title{
Is the ovarian reserve influenced by vitamin $D$ deficiency and the dress code in an infertile Iranian population?
}

Soheila Arefi ${ }^{1,2,3}$, Gholamreza Khalili', Homa Iranmanesh ${ }^{5}$, Fattaneh Farifteh ${ }^{6}$, Ahmad Hosseini ${ }^{6}$, Human M. Fatemi ${ }^{7}$ and Barbara Lawrenz ${ }^{7,8^{*}}$ (i)

\begin{abstract}
Background: In the recent years, vitamin D has become a topical subject and a focus of research not only in reproductive medicine but across many medical disciplines. In reproductive medicine, studies have identified an association between vitamin D status in women and ovarian reserve. In humans, exposure of the skin to sunlight is the main important source of vitamin D. A dress code of wearing concealing clothing is a risk factor for vitamin $\mathrm{D}$ deficiency. The objective of this prospective observational study was to evaluate the correlation between vitamin $\mathrm{D}$ deficiency and ovarian reserve in a population of infertile women in Iran. As part of the basic fertility assessment of study participants, blood tests were taken to measure vitamin D concentration and transvaginal ultrasound scans were performed on day 2 -5 of the cycle to determine antral follicle count (AFC). All study participants were assessed by a reproductive medicine specialist and consultant dermatologist to classify their skin types according to the Fitzpatrick classification. In addition, the dress code of each study participant was recorded noting the percentage of exposed skin not covered by concealing clothing.

Results: 189 infertility patients were included in this study. The mean concentration of vitamin D in this study population was $15.46 \mathrm{ng} / \mathrm{ml}$, indicating severe vitamin D deficiency. A statistically significant negative correlation between age and vitamin $D(p=0.008)$ and age and AFC ( $p=0.001)$ was identified. This study revealed a highly significant correlation between vitamin D concentrations and AFC $(p<0.001)$.

Conclusions: A concealing dress code is an independent risk factor for vitamin D deficiency due to a lack of skin exposure to sunlight. Our study suggests that the so caused severe vitamin D deficiency may play a crucial role in reduced ovarian reserve in the herein described group of an infertile female Iranian population.
\end{abstract}

Keywords: Dress code, Vitamin D deficiency, Ovarian reserve, Skin-type

\section{Background}

In recent years, vitamin $\mathrm{D}$ has become a topical subject and a focus of research in several medical disciplines.

Vitamin D is widely recognized to facilitate absorption of calcium and promote bone growth. Vitamin D deficiency in children may result in a condition known as rickets incurring an increased risk of fractures and deformity. In adults, vitamin D deficiency may result in

\footnotetext{
*Correspondence: barbara.lawrenz@ivirma.com

${ }^{7}$ IVF department, IVI RMA Middle-East Fertility Clinic, Abu Dhabi, UAE

${ }^{8}$ Women's university hospital Tuebingen, Tuebingen, Germany

Full list of author information is available at the end of the article
}

osteomalacia carrying the same risks of fracture and deformity. In addition to its role in bone health, it appears that vitamin D deficiency is also involved in the pathogenesis of many chronic diseases such as autoimmune diseases, inflammatory bowel disease, infections, immune deficiency, cardiovascular diseases, cancer and neurocognitive disorders [1].

As vitamin D receptors are widely found in reproductive tissues, including the ovaries and the endometrium, an important role of vitamin D in human reproduction has been suggested. Vitamin D status has been suggested to be associated with ovarian reserve and with the

(c) The Author(s). 2018 Open Access This article is distributed under the terms of the Creative Commons Attribution 4.0 International License (http://creativecommons.org/licenses/by/4.0/), which permits unrestricted use, distribution, and reproduction in any medium, provided you give appropriate credit to the original author(s) and the source, provide a link to the Creative Commons license, and indicate if changes were made. The Creative Commons Public Domain Dedication waiver (http://creativecommons.org/publicdomain/zero/1.0/) applies to the data made available in this article, unless otherwise stated. 
outcome of in vitro fertilization (IVF) treatments as well as with features of polycystic ovarian syndrome (PCOS) and endometriosis. In men, associations with semen quality and sperm count, motility and morphology had been described [2].

Vitamin D belongs to a group of fat-soluble vitamins and is responsible for the intestinal absorption of calcium, magnesium, phosphate, zinc and iron. The most important forms of Vitamin D are vitamin D3 (cholecalciferol) and vitamin D2 (ergocalciferol) [3]. According to recent studies, vitamin $\mathrm{D}$ deficiency is defined as serum-levels of $25(\mathrm{OH}) \mathrm{D}$ below $20 \mathrm{ng} / \mathrm{ml}$. The importance of vitamin $\mathrm{D}$ deficiency is underlined by the fact, that an estimated 1 billion people worldwide are vitamin $\mathrm{D}$ deficient and deficiencies can be found in all ethnicities and age groups [4]. Therefore vitamin D deficiency is the most common vitamin deficiency worldwide in both, children and adults [5].

Dietary sources of vitamin D alone are not sufficient to maintain normal vitamin D levels.

The most important source of vitamin $\mathrm{D}$ for humans is exposure to sunlight, particularly ultraviolet B rays. Several factors influence vitamin D production following sun exposure. These factors include age, skin color, degree of exposed skin, length of time exposed to sunlight, geographic location, time of year, time of day, cloud cover, smog, dust or haze.

The countries of the Middle East have the highest vitamin D deficiency rates in the world, ranging from $67 \%$ in Iran, $55-83 \%$ in Jordan, $84 \%$ in Lebanon up to $90 \%$ in Saudi Arabia [6].

Recently, several studies evaluated the influence of vitamin $\mathrm{D}$ on the ovarian reserve [7-9]. In contrast to the herein presented study, the participants consisted mainly of women of Caucasian ethnicity and were not subjected to imposed female dress codes. Our study is unique in that our participants are of Iranian ethnicity and wear concealing clothing, a dress code imposed by both, religious and social values.

The aim of this study was to evaluate the vitamin D status in an infertile population in Iran and to correlate vitamin D concentrations with parameters such as female age, skin type, degree of skin concealment and antral follicle count (AFC) as parameter of the ovarian reserve.

\section{Methods}

This observational prospective study was performed in 2 independent private infertility centers in Iran. Patients, referred to the fertility centers with both, primary and secondary infertility, were considered eligible for inclusion in the study. Exclusion criteria included any factors which may adversely affect ovarian reserve such as a history of endometriosis, history of previous pelvic surgery, smokers and recreational drug users, history of chemotherapy or pelvic radiation and a history of hormonal treatment in the previous 6 months. Polycystic ovarian syndrome as defined by the Rotterdam-criteria [10] was also included as an indication for exclusion from the study. The intake of calcium, vitamin D or multivitamin supplements in the previous 3 months or a history of poor ovarian response according to the Bologna criteria [11] in a previous ART-treatment were also exclusion factors.

All study participants signed an informed consent agreement. Blood for vitamin D concentrations were measured via venipuncture and was taken together with other blood tests, deemed necessary for evaluation of the existing infertility and further treatment planning. Due to the additional costs which would have been created by performing $\mathrm{AMH}$-testing and which would have to be covered by the patients, no routine AMH-test was performed. Transvaginal 2D ultrasound of the pelvis was performed on day 2-5 of the cycle to exclude pelvic pathology and to determine AFC.

\section{Ultrasound for antral follicle count measurement}

All patients were scanned by transvaginal 2D ultrasound on day $2-5$ of the cycle. The ultrasound-scans were performed by one designated reproductive medicine specialist in each center in order to reduce the inter-observer variability. The two involved investigators established a common ultrasound technique for the AFC measurement prior of commencing the study in order to avoid any bias through different techniques.

All study participants had a transvaginal scan performed by the same investigator for intra-observer or inter-observer reliability, using a vaginal probe (Honda 5-9 MHZ, HS-2600, Japan). The optimal probe program, providing the best ultrasound image, was used in both fertility centers and the settings were maintained throughout the duration of the ultrasound evaluation. Each patient was asked to empty the bladder and was scanned in a Lloyd Davies position to ensure free manipulation of the transvaginal transducer. Ultrasound assessment of the pelvis excluded pelvic pathology. The ovaries were visualized in the longitudinal plane and the number of antral follicles measuring $2-10 \mathrm{~mm}$ in diameter within each ovary was counted as the transducer was moved from one side of the ovary to the other. Two scans were obtained for each ovary and in case of any discrepancy between these two values, a third measurement was done to confirm the final value.

\section{Skin-type}

The skin-types of all study participants was evaluated and classified according to the different skin type groups 
described by the Fitzpatrick classification [12] by a reproductive specialist and a consultant dermatologist.

Type I-II Fitzpatrick classification - abbreviated in this study as F1:

- Type I (scores 0-6) always burns, never tans (pale white; blond or red hair; blue eyes; freckles)

- Type II (scores 7-13) usually burns, tans minimally (white; fair; blond or red hair; blue, green, or hazel eyes)

Type III - IV 3-4 Fitzpatrick classification - abbreviated in this study as F2:

- Type III (scores 14-20) sometimes mild burn, tans uniformly (cream white; fair with any hair or eye color)

- Type IV (scores 21-27) burns minimally, always tans well (moderate brown)

Type V - Fitzpatrick classification - abbreviated in this study as F3:

- Type V (scores 28-34) very rarely burns, tans very easily (dark brown)

\section{Dress-code}

According to the Iranian regulations, in any public place women must cover their heads with a headscarf, wear trousers (or a floor length skirt), and a long-sleeved tunic or coat that reaches to mid-thigh or knee. Depending on the amount of skin not covered by concealing cloths, patients were stratified into the dress code (DR) 1, 2 and 3. Patients with DR 1 had an amount of uncovered skin of 10 to $15 \%$, patients in DR 2 between 5 and $10 \%$ and patients in group DR 3 below 5\% of skin exposed to sunlight.

\section{Measurement of vitamin D-levels}

Blood samples were taken to measure vitamin D concentrations within the first week following the initial consultation with a reproductive medicine specialist and at the same time of ultrasonographic assessment of the antral follicle count (AFC). Once the blood sample was taken for vitamin D concentration, the sample was centrifuged and frozen at $-20^{\circ}$ Celsius. All blood samples for vitamin $\mathrm{D}$ concentrations were measured by the ELISA assay at one time to avoid inter-assay variability, using electrochemiluminescence binding assay (Roche's test) and Cobas e immunoassay analyzers.

Vitamin D deficiency was defined as serum level of < $20 \mathrm{ng} / \mathrm{ml}$ 25hydroxy vitamin D [3].

\section{Statistical analysis}

Data were analyzed by SPSS v.18 software (IBM, Chicago, IL, USA). The quantitative measurements were described by using the mean and the standard deviation (SD). For nominal variables the relative frequency, using percentages, were reported. For comparison of means among groups, analysis of variance and LSD post hoc test was used.

\section{Results}

A total of 189 patients have been included in this prospective observational study. $87.7 \%(n=166)$ of the patients attended the clinics due to primary infertility. The mean BMI of the patients was $26.70 \mathrm{~kg} / \mathrm{m}^{2}$ with a range of $17.9-35.5 \mathrm{~kg} / \mathrm{m}^{2}$.

According to the Fitzpatrick classifications, 50 patients (26.4\%) were categorized as Fitzpatrick I-II (F1), 89 (47.1\%) as Fitzpatrick III-IV (F2) and 50 (26.4\%) as Fitzpatrick V (F3).

The patients were also classified according to dress code DR1, DR2 and DR 3 as previously described with the following distribution, $110(58.2 \%), 30(15.9 \%)$ and $49(25.9 \%)$ respectively.

The mean age of the study group was 32.21 years and for the groups F1, F2 and F3 31.76 years, 32.40 years and 32.32 years respectively. The mean age of the patients in the different groups classified according to dress-code groups were as follows: DR1: 31.97 years, DR2: 33.07 years and DR3: 32.22 years.

The mean vitamin D level for the total study group was $15.46 \mathrm{ng} / \mathrm{ml}$. In group F1 mean vitamin D level was $16.39 \mathrm{ng} / \mathrm{ml}$, in group F2 $14.50 \mathrm{ng} / \mathrm{ml}$ and in group F3 $16.23 \mathrm{ng} / \mathrm{ml}$. The mean Vitamin D level for groups DR1, DR2 and DR3 were $16.15 \mathrm{ng} / \mathrm{ml}, 13.66 \mathrm{ng} / \mathrm{ml}$ and $14.21 \mathrm{ng} / \mathrm{ml}$, respectively.

The mean AFC count in all patients was 10.84. Patients in group $\mathrm{F} 1$ had a mean AFC of 10.14, group F2 a mean AFC of 11.20 and group F3 a mean AFC of 10.90. In the dress-code groups the results were: DR1: 10.59, DR2: 9.87 and DR3: 12.00, respectively. Table 1 provides the summary of the results.

The correlation of the data of all study patients between the parameters age, vitamin D concentrations and AFC showed a significant negative correlation between age and vitamin D $(p=0.008)$, age and AFC $(p=0.001)$ and importantly, a highly statistically significant correlation between vitamin D and $\operatorname{AFC}(p<0.001)$.

No significant differences were found between and within the different skin-type groups for age $(p=0.59)$, vitamin $\mathrm{D}(p=0.56)$ and AFC $(p=0.66)$. The groups of different dress-codes were also found to have no significant differences between and within the group for the parameters age $(p=0.34)$, Vitamin $\mathrm{D}(p=0.32)$ and $\operatorname{AFC}(p$ $=0.31$ ). Also no correlations were found between vitamin 
Table 1 Summary of the results for the means, ranges and standard deviations for the following parameters: age, vitamin D concentrations and AFC in the different skin-type- and dress-code-groups

\begin{tabular}{|c|c|c|c|c|c|c|c|c|c|}
\hline \multicolumn{2}{|l|}{ Groups } & $\begin{array}{l}\text { Total } \\
\text { group }\end{array}$ & F1 & F2 & F3 & DR1 & DR2 & DR3 & Significance \\
\hline \multicolumn{2}{|c|}{ Number of patients and \% } & 189 (100\%) & $\begin{array}{l}50 \\
(26.4 \%)\end{array}$ & $\begin{array}{l}89 \\
(47.1 \%)\end{array}$ & $\begin{array}{l}50 \\
(26.4 \%)\end{array}$ & $\begin{array}{l}110 \\
(58.2 \%)\end{array}$ & $\begin{array}{l}30 \\
(15.9 \%)\end{array}$ & $\begin{array}{l}49 \\
(25.9 \%)\end{array}$ & n.a. \\
\hline \multirow[t]{3}{*}{ Age (years) } & Mean & 32.21 & 31.76 & 32.4 & 32.32 & 31.97 & 33.07 & 32.22 & n.s. \\
\hline & Range & $21-42$ & $21-38$ & $23-43$ & $21-38$ & $21-39$ & $27-42$ & $26-40$ & \\
\hline & SD & 3.64 & 3.63 & 3.69 & 3.58 & 3.67 & 3.83 & 3.44 & \\
\hline \multirow[t]{3}{*}{$\begin{array}{l}\text { Vitamin D level (ng/ } \\
\text { ml) }\end{array}$} & $\begin{array}{l}\text { Mean Vit. D level (ng/ } \\
\text { ml) }\end{array}$ & 15.46 & 16.39 & 14.50 & 16.23 & 16.15 & 13.66 & 14.21 & n.s. \\
\hline & Range & $2.5-73$ & $2.5-43.8$ & $2.5-73$ & $4-48$ & $2.5-73$ & $2.5-48$ & $4.0-44$ & \\
\hline & SD & 11.5 & 11.01 & 11.32 & 11.05 & 11.91 & 10.54 & 11.08 & \\
\hline \multirow[t]{3}{*}{ AFC } & Mean AFC & 10.84 & 10.14 & 11.20 & 10.90 & 10.59 & 9.87 & 12.00 & n.s. \\
\hline & Range & $1-31$ & $2-29$ & $2-31$ & $1-30$ & $1-31$ & $2-29$ & $2-29$ & \\
\hline & SD & 6.63 & 6.45 & 7.06 & 6.06 & 6.43 & 7.54 & 6.37 & \\
\hline
\end{tabular}

n.a. not applicable, n.s. not significan, SD Standard deviation

$\mathrm{D}$ and BMI $(p=0.968)$ and vitamin D and the type of infertility ( $p=0.451)$. The performance of multiple comparisons in the groups age, vitamin D and AFC within the different skin types as well as within the different dress codes did not show any significant differences.

\section{Discussion}

The presence of vitamin $\mathrm{D}$ receptors in reproductive tissues $[13,14]$ and the fact that in animal studies impaired folliculogenesis [15] was found in rats with compromised expression of vitamin $D$ receptors suggests, that vitamin $\mathrm{D}$ deficiency may play an important role in determining the ovarian reserve. It was previously described that 1.25-dihydroxyvitamin D3 alters Anti-Mullerian-Hormone (AMH) sensitivity in human granulosa cells by inhibiting the expression of the $\mathrm{AMH}$ receptor (AMHR-II). Due to a reduced AMH sensitivity, more follicle might reach terminal maturation and ovulation, thereby influencing the ovarian reserve [16]. This possible interaction between the ovarian reserve and vitamin $\mathrm{D}$ is underlined by a correlation of seasonal fluctuations in serum AMH with seasonal changes in the vitamin D levels [17]. Due to this correlation, Irani et al. [7] even considered vitamin D to be a clinically useful marker of the ovarian reserve and suggested to include vitamin $\mathrm{D}$ into the routine workup in patients with infertility.

The herein presented study findings result from a prospective observational study in 189 female infertility patients in Iran, who are obliged to wear a specific dress code. The results demonstrate clearly a highly significant correlation $(p<0.001)$ between vitamin D deficiency and a reduced ovarian reserve. In this setting of self-payer patients, ovarian reserve was assessed by the antral follicle count (AFC), which is an established parameter to evaluate the ovarian reserve [18].

The most commonly used parameters for ovarian reserve testing are FSH (Follicle-stimulating-hormone), $\mathrm{AMH}$ (Anti-Muellerian-Hormone) and the antral follicle count. Nowadays, FSH is regarded as a parameter with poor sensitivity towards the detection of a diminished ovarian reserve and FSH testing has several major limitations including significant inter-cycle and intra-cycle variability and elevated FSH levels are almost only found in patients with an existing diminished ovarian reserve [19]. AMH is produced by the granulosa cells of small and large preantral and small antral follicles, therefore reflecting the primordial follicle pool. Whereas it was suggested that $\mathrm{AMH}$ is relatively stable throughout the cycle [20,21], recent publications demonstrated significant fluctuations within a menstrual cycle $[22,23]$. The antral follicle count (AFC) is calculated as the sum of follicles in both ovaries in the early follicular phase (day 2-4) of the menstrual cycle. The assessment can be performed in the context of the primary ultrasound evaluation of the internal genital organs and it provides an immediate result, has a good inter-cycle reliability and, if performed by experienced and a limited number of investigators, a good inter-observer reliability [24]. Several studies have shown that AFC and AMH are both good predictors of the ovarian response in the context of hormonal stimulation for ART (assisted reproductive techniques) treatment $[25,26]$, with each method having special advantages, disadvantages and limitations.

Studies to date, investigating the role of vitamin D on the ovarian reserve are conflicting and our results are in contrast to the findings from the recently published studies of Fabris et al. [8] and Drakopoulos et al. [9] who could not find a correlation between the ovarian reserve parameters AFC / AMH and vitamin D deficiency. 
The contradictory finding between the studies may be attributed to the different ethnicities of the study population, having different socio-cultural religious habits and different dress-codes. Drakopoulos et al. [9] evaluated women with Caucasian ethnicity, who have been treated in an infertility center due to primary / secondary infertility in Belgium. The study of Fabris et al. [8] included only oocyte donors, who are usually young and healthy and don't have any infertility problems. The ethnicity of the study population is not revealed. However, their data are derived from an oocyte-donation-program based at a Spanish IVF-clinic. Therefore it can be assumed that their study patients had Caucasian ethnicity as well. In contrast to those mentioned studies, the herein described study population presents firstly a completely different ethnicity and secondly around $40 \%$ of the study population is dressed in a dress-code, which leaves less than $90 \%$ of the skin uncovered, thereby limiting the skin-exposure to the sun dramatically.

The Middle East countries have the highest vitamin D deficiency rates in the world and different factors contribute to low sun exposure and low vitamin D levels in women of reproductive age in these countries [27]. One of the most important factors leading to a high rate of vitamin $\mathrm{D}$ deficiency is the dress code of wearing concealing clothing due to social and religious habits [28]. The concealing dress code has been found to be an independent prognostic factor of vitamin D deficiency in Lebanese women, confirming the significance of sunlight exposure in vitamin D synthesis [28] and differences observed in vitamin D deficiency rates between Christian and Muslim women in Middle East highlight the importance of the dress code [29].

Skin-type is an important factor in determining the time needed for sufficient vitamin D synthesis via sunlight exposure. Persons with a skin-type classification I or II achieve maximal vitamin $\mathrm{D}$ photosynthesis rapidly after approximately 2-8 min of midday spring or summer sun exposure in New York or Boston and only slightly longer in Alaska or Scandinavia. Persons with phototype III skin produce vitamin $\mathrm{D}$ at similar rates to persons with phototype I or II skin after a first UV exposure. Persons with skin-type IV have high epidermal melanin content and therefore photosynthesize relatively limited amounts of vitamin D because of UV absorption by melanin [30]. Other authors deem the exposure of arms and legs for 5 to $30 \mathrm{~min}$ between 10 a.m. and 3 p.m. twice a week for sufficient to achieve adequate vitamin D levels [31].

However, in most Middle-East Islamic populations, girls start to wear concealing clothing with the onset of puberty or even earlier, leading to long-lasting non-exposure of the skin to the sun and therefore to long-lasting Vitamin D deficiencies.

Evaluation of the influence of the skin-type and dress-code on the Vitamin D deficiency was performed in a subgroup-analysis and no significant correlations between the different skin-types / dress-codes and vitamin D deficiency were found. Due to wearing concealing clothing with coverage of most of the body surface, the skin-type is of negligible importance. Absence of a correlation between the different dress-codes and vitamin $\mathrm{D}$ deficiency is explained by the fact, that even the group with the least skin coverage (10-15\% at most of body skin surface which could be exposed to the sun) is still far below the required $20 \%$.

Our study population is characterized by a severe vitamin D deficiency with $71.4 \%$ (135 patients) of the patients being vitamin $\mathrm{D}$ deficient and a mean vitamin $\mathrm{D}$ level in the study group of $15.48 \mathrm{ng} / \mathrm{ml}$. In contrast, the mean vitamin $D$ levels are not mentioned in the studies of Drakopoulos and Fabris [8, 9]. In addition, less than $50 \%$ of their study populations were vitamin D deficient (30 and 18\% respectively). Therefore the severity of vitamin D deficiency in their studies, when compared to our study, was less and may be the reason why no correlation between vitamin $\mathrm{D}$ deficiency and the ovarian reserve parameters was identified.

\section{Conclusions}

The herein presented data may indicate, that severe and long-lasting vitamin D deficiency, caused by inadequate sun-exposure to the skin, could be a cause of the reduced ovarian reserve in the herein investigated group of infertile female Iranian patients. Future studies are required to investigate further the interaction between dress-code, vitamin D and fertility.

\section{Acknowledgements}

We thank Dr. Carol Coughlan for the linguistic revision and correction of the manuscript.

Availability of data and materials

All data is presented in the paper.

Authors' contributions

SA Conceptualization: Lead; Supervision: Lead; Investigation: Lead; Methodology: Lead; Writing - review \& editing: Supporting. GK Conceptualization: Supporting; Formal analysis: Lead; Investigation: Equal; Writing - review \& editing: Supporting. HI Conceptualization: Supporting; Investigation: equal. FF Conceptualization: Supporting; Investigation: equal. AH Conceptualization: Supporting; Formal analysis: lead; Investigation: equal. HMF Conceptualization: Supporting; Supervision: equal; Writing - review editing: equal. BL formal analysis: Supporting; writing- original draft: Lead. All authors read and approved the final manuscript.

Ethics approval and consent to participate

This prospective observational study had been approved by the Ethical Committee of Shahid Beheshti University, Cellular and molecular biology research center, Iran, in 2015. The trial registration number is IR.SBMU.RETECH.REC.1395.285.

Consent for publication

All authors agree with the submission of this paper. 


\section{Competing interests}

Soheila Arefi, Gholamreza Khalili, Homa Iranmanesh, Fattaneh Farifheh, Ahmad Hosseini, Human M. Fatemi and Barbara Lawrenz have no competing interests.

\section{Publisher's Note}

Springer Nature remains neutral with regard to jurisdictional claims in published maps and institutional affiliations.

\section{Author details}

'Avicenna Research Institute, ACECR, Tehran, Iran. ${ }^{2}$ Bahman Hospital Infertility Center, North Iran Zamin St, Shahrak Gharb, Tehran, Iran. ${ }^{3}$ Givar Infertility Center, Tehran, Iran. ${ }^{4}$ Department of Epidemiology and Reproductive Health, Reproductive Research Center, Royan Institute for Reproductive Biomedicine, ACECR, Tehran, Iran. ${ }^{5}$ Sarem Infertility Center, Sarem Women's Hospital, Shahrak Ekbatan, Tehran, Iran. ${ }^{6}$ Cellular and Molecular Biology Research Center, Shahid Beheshti University of Medical science, Arabi Ave, Daneshjoo Blvd, Velenjak, Tehran, Iran. ${ }^{7}$ IVF department, IVI RMA Middle-East Fertility Clinic, Abu Dhabi, UAE. ${ }^{8}$ Women's university hospital Tuebingen, Tuebingen, Germany.

Received: 7 April 2018 Accepted: 19 July 2018

Published online: 24 July 2018

\section{References}

1. Groeber U, Spitz J, Reichrath J, Kisters K, Holick MF. Vitamin D update 2013: from rickets prophylaxis to general preventive healthcare. Dermatoendocrinol. 2013;5(3):331-47.

2. Anagnostis $P$, Karras $S$, Goulis DG. Vitamin D in human reproduction: a narrative review. Int J Clin Pract. 2013;67(3):225-35.

3. Holick MF. Vitamin D deficiency. N Engl J Med. 2007;357(3):266-81.

4. Lips P. Worldwide status of vitamin D nutrition. J Steroid Biochem Mol Biol. 2010;121:297-300.

5. Hossein-Nezhad A, Holick MF. Vitamin D for health: a global perspective. Mayo Clin Proc. 2013;88(7):720-55.

6. Lips P. Vitamin D status and nutrition in Europe and Asia. J Steroid Biochem Mol Biol. 2007:103(3-5):620-5.

7. Irani M, Merhi Z. Role of vitamin D in ovarian physiology and its implication in reproduction: a systematic review. Fertil Steril. 2014;102:460-8.

8. Fabris AM, Cruz M, Iglesias C, Pacheco A, Patel A, Patel J, et al. Impact of vitamin $\mathrm{D}$ levels on ovarian reserve and ovarian response to ovarian stimulation in oocyte donors. Reprod BioMed Online. 2017;35(2):139-44.

9. Drakopoulos P, van de Vijver A, Schutyser V, Milatovic S, Anckaert E, Schiettecatte J, et al. The effect of serum vitamin D levels on ovarian reserve markers: a prospective cross-sectional study. Hum Reprod. 2017;32(1):208-14.

10. The Rotterdam ESHRE/ASRM-Sponsored PCOS Consensus Workshop Group. Revised 2003 consensus on diagnostic criteria and long-term health risks related to polycystic ovary syndrome (PCOS). Hum Reprod. 2004;19:41-7.

11. Ferraretti AP, La Marca A, Fauser BC, Tarlatzis B, Nargund G, Gianaroli L. ESHRE working group on poor ovarian response definition. ESHRE consensus on the definition of 'poor response' to ovarian stimulation for in vitro fertilization: the bologna criteria. Hum Reprod. 2011;26(7):1616-24.

12. Fitzpatrick TB. The validity and practicality of sun-reactive skin types I through VI. Arch Dermatol. 1988 Jun;124(6):869-71.

13. Shahbazi M, Jeddi-Tehrani M, Zareie M, Salek-Moghaddam A, Akhondi MM, Bahmanpoor $\mathrm{M}$, et al. Expression profiling of vitamin D receptor in placenta, decidua and ovary of pregnant mice. Placenta. 2011;32:657-64.

14. Zarnani AH, Shahbazi M, Salek-Moghaddam A, Zareie M, Tavakoli M, Ghasemi J, et al. Vitamin D3 receptor expressed in the endometrium of cycling mice throughout the estrous cycle. Fertil Steril. 2010;93:2738-43.

15. Halloran BP, DeLuca HF. Effect of vitamin D deficiency on fertility and reproductive capacity in the female rat. J Nutr. 1980;110:1573-80.

16. Merhi Z, Doswell AD, Krebs K, Cipolla MJ. Vitamin D alters genes involved in follicular development and steroidogenesis in human cumulus granulosa cells. J Clin Endocrinol Metab. 2014;14:jc20134161.

17. Dennis NA, Houghton LA, Jones GT, van RijAM MK, McLennan IS. The leve of serum anti-mullerian hormone correlates with vitamin D status in men and women but not in boys. J Clin Endocrinol Metab. 2012;97:2450-5.

18. Fleming R, Seifer DB, Frattarelli $J$, Ruman J. Assessing ovarian response: antral follicle count versus anti-Müllerian hormone. Reprod BioMed Online 2015;31(4):486-96.
19. Broekmans FJ, Kwee J, Hendriks DJ, Mol BW, Lambalk CB. A systematic review of tests predicting ovarian reserve and IVF outcome. Hum Reprod Update. 2006;12(6):685-718.

20. Hehenkamp WJ, Looman CW, Themmen AP, de Jong FH, Te Velde ER, Broekmans FJ. Anti-Mullerian hormone levels in the spontaneous menstrual cycle do not show substantial fluctuation. J Clin Endocrinol Metab. 2006; 91(10):4057-63.

21. La Marca A, Stabile G, Artenisio AC, Volpe A. Serum anti-Mullerian hormone throughout the human menstrual cycle. Hum Reprod. 2006;21(12):3103-7.

22. Kissell KA, Danaher MR, Schisterman EF, Wactawski-Wende J, Ahrens KA, Schliep K, et al. Biological variability in serum anti-Mullerian hormone throughout the menstrual cycle in ovulatory and sporadic anovulatory cycles in eumenorrheic women. Hum Reprod. 2014;29(8):1764-72.

23. Hadlow N, Longhurst K, McClements A, Natalwala J, Brown SJ, Matson PL. Variation in antimullerian hormone concentration during the menstrual cycle may change the clinical classification of the ovarian response. Fertil Steril. 2013;99(6):1791-7.

24. Tal R, Seifer DB. Ovarian reserve testing: a user's guide. Am J Obstet Gynecol. 2017;217(2):129-40.

25. La Marca A, Sighinolfi G, Radi D, Argento C, Baraldi E, Artenisio AC, Stabile G, Volpe A. Anti-Mullerian hormone (AMH) as a predictive marker in assisted reproductive technology (ART). Hum Reprod Update. 2010;16(2):113-30.

26. Lukaszuk K, Kunicki M, Liss J, Lukaszuk M, Jakiel G. Use of ovarian reserve parameters for predicting live births in women undergoing in vitro fertilization. Eur J Obstet Gynecol Reprod Biol. 2013;168(2):173-7.

27. Mishal AA. Effects of different dress styles on vitamin D levels in healthy young Jordanian women. Osteoporos Int. 2001;12(11):931-5.

28. Hatun S, Islam O, Cizmecioglu F, Kara B, Babaoglu K, Berk F, et al. Subclinical vitamin $D$ deficiency is increased in adolescent girls who wear concealing clothing. J Nutr. 2005;135(2):218-22.

29. Gannagé-Yared MH, Maalouf G, Khalife S, Challita S, Yaghi Y, Ziade N, et al. Prevalence and predictors of vitamin D inadequacy amongst Lebanese osteoporotic women. Br J Nutr. 2009;101(4):487-91.

30. Gilchrest BA. Sun exposure and vitamin D sufficiency. Am J Clin Nutr. 2008; 88(2):570S-7S.

31. Holick MF. Vitamin D: the underappreciated D-lightful hormone that is important for skeletal and cellular health. Curr Opin Endocrinol Diabetes. 2002;9:87-98
Ready to submit your research? Choose BMC and benefit from:
- fast, convenient online submission
- thorough peer review by experienced researchers in your field
- rapid publication on acceptance
- support for research data, including large and complex data types
- gold Open Access which fosters wider collaboration and increased citations
- maximum visibility for your research: over $100 \mathrm{M}$ website views per year
At BMC, research is always in progress.
Learn more biomedcentral.com/submissions 